\title{
Experimental generation of optical flaticon pulses
}

\author{
Bastien Varlot, ${ }^{1}$ Stefan Wabnitz, ${ }^{2}$ Julien Fatome, ${ }^{1}$ Guy Millot, ${ }^{1}$ and Christophe Finot ${ }^{1, *}$ \\ ${ }^{1}$ Laboratoire Interdisciplinaire Carnot de Bourgogne (ICB), UMR 6303 CNRS/Université de Bourgogne, Dijon, France \\ ${ }^{2}$ Department of Information Engineering, Università di Brescia, Brescia, Italy \\ ${ }^{*}$ Corresponding author: christophe.finot@u-bourgogne.fr
}

Received July 15, 2013; accepted August 29, 2013;

posted September 3, 2013 (Doc. ID 193952); published September 26, 2013

\begin{abstract}
We experimentally investigate the nonlinear reshaping of a continuous wave that leads to chirp-free and flat-top intense pulses or flaticons exhibiting strong temporal oscillations at their edges and a stable self-similar expansion upon propagation of their central region. This study was performed in the normal dispersion regime of a nonzero dispersion-shifted fiber and involved a sinusoidal phase modulation of the continuous wave. Our fiber optics experiment is analogous to considering the collision between oppositely directed currents near the beach, and it may open the way to new investigations in the field of hydrodynamics. (c) 2013 Optical Society of America OCIS codes: (060.4370) Nonlinear optics, fibers; (190.4380) Nonlinear optics, four-wave mixing. http://dx.doi.org/10.1364/OL.38.003899
\end{abstract}

Since the birth of nonlinear optics, there has frequently been a cross-fertilization with hydrodynamics in the study of nonlinear wave propagation phenomena. Breakers on a sloping beach and river bores [1,2] are analogous to optical wave breaking and shocks that appear in the normal group velocity dispersion (GVD) regime of optical fibers [3]. Self-filamentation of light beams []ㅡ ] has the same origin as wave train disintegration in deep water [5]. The classical dam break problem [6] also describes the distortion of nonreturn-to-zero optical pulses in long distance fiber optics communications [7]. Dark soliton pulses have been observed in optical fibers since a long time [8], and just recently in surface water waves as well [9]. Extreme water waves, often known as freak or rogue waves, have been known for quite some time in oceanography [10]. Yet, the first experiments demonstrating the generation of a prototype rogue wave, known as the Peregrine soliton [11], have been performed using standard telecommunications nonlinear optical fibers and components [12]. Extreme waves are also well known to occur in shallow waters, e.g., the run-up of a tsunami toward the coast [13]. Once again, similar phenomena have been recently predicted to occur in nonlinear optical fibers with normal GVD [14,15].

In shallow water, the crossing of currents that propagate with opposite directions may lead to the formation of high-elevation and steep humps of water (or sneaker waves) that could result in severe coastal damages. The analogous effect may occur in optical fibers: In $[14,16]$ it was numerically and analytically shown that a continuous-wave (CW) light beam subject to an initial step-wise periodic frequency modulation evolves, upon propagation in a fiber with normal GVD, toward a train of intense, stable, and chirp-free optical pulses. In this case, the physical mechanism leading to pulse formation is the collision among the slower, positively chirped leading wavefront with the faster, negatively chirped trailing wavefront. Given the high degree of flatness of these nonlinear structures, we name them flaticons. Quite remarkably and as predicted in [16], flaticons experience a stable self-similar evolution, undergoing a linear expansion of their temporal width while maintaining their peakpower constant.
In the present contribution, we describe the experimental generation of optical flaticons in optical fibers at telecommunication wavelengths, by exploiting the nonlinear and dispersive reshaping of an intense CW in the presence of a suitable input sinusoidal phase modulation [14]. The Letter is therefore organized as follows. We first recall the theoretical background of flaticon pulse generation. We then describe the details of the experimental setup that we implemented in order to first imprint the required sinusoidal phase modulation on the initial $\mathrm{CW}$, and then to observe the subsequent nonlinear reshaping upon propagation into a normally dispersive optical fiber. Finally, we discuss the influence of both the input CW power and the initial phase modulation amplitude (or chirp), and confirm the chirp-free nature of the central part of the flaticon.

Propagation of light in optical fibers is described by the nonlinear Schrödinger equation (NLSE)

$$
i \frac{\partial \psi}{\partial z}-\frac{\beta_{2}}{2} \frac{\partial^{2} \psi}{\partial t^{2}}+\gamma|\psi|^{2} \psi+i \frac{\alpha}{2} \psi=0,
$$

where $z$ and $t$ denote the propagation distance and the retarded time (in the frame traveling at the groupvelocity) coordinates; $\beta_{2}$ and $\gamma$ are the second-order GVD and the nonlinear Kerr coefficient of the fiber, respectively, and $\psi(t, z)$ is the complex field envelope. Fiber losses $\alpha$ have been included in order to get a better agreement with the experiments; however their low level has no qualitative influence on the nonlinear dynamics under investigation. [14] analyzed the progressive reshaping in a fiber with normal GVD of a CW of power $P$ with a negative input frequency jump. In this case, it has been shown that, whenever the dispersion length is large with respect to the nonlinear length (i.e., in the semiclassical limit of the NLSE [2,14]), optical pulse dynamics can be described in terms of the nonlinear shallow water equation. In hydrodynamics, this equation is well known to describe water wave propagation in rivers or near the coastline: In that context, the optical intensity and instantaneous frequency translate into the water depth and its velocity. In such a framework, the previously described initial value problem has an 
analytical solution. It corresponds to the generation of a so-called flaticon pulse, which may stably propagate whenever the negative frequency jump remains below a critical value $f_{c}$, that reads in dimensional units as $f_{c}=\sqrt{\gamma P / \beta_{2}} / \pi[\underline{14}]$. The case of an initial sinusoidal phase modulation $\varphi(t)=\varphi_{M} \sin \left(\omega_{0} t\right)$, with $\varphi_{M}$ and $\omega_{0}$ being the amplitude and the frequency of the modulation, respectively, has also been discussed in [14]. In this case it has been pointed out that, as long as the total frequency jump $f_{s}=2 \varphi_{M} \omega_{0}$ does not exceed $2.4 f_{c}$, a flaticon-like structure should also appear.

The experimental setup for demonstrating flaticon generation is depicted in Fig. 1(a), and it relies exclusively on commercially available components ready for telecommunication use. The setup can be divided into two parts.

The first part is aimed to imprint on a CW signal the required phase modulation. In order to obtain a modulation amplitude well above the $\pi$ radian value that is routinely provided by phase modulators for telecommunication applications, we took advantage of the phenomenon of cross-phase modulation (XPM) imposed by an intensity modulated pump wave upon a CW seed in a $1 \mathrm{~km}$ long highly nonlinear optical fiber (HNLF) with low normal dispersion. The pump wave at $1550 \mathrm{~nm}$ is thus modulated by an intensity modulator driven by a sinusoidal electrical clock running at $12.5 \mathrm{GHz}$. Care has been

(a)
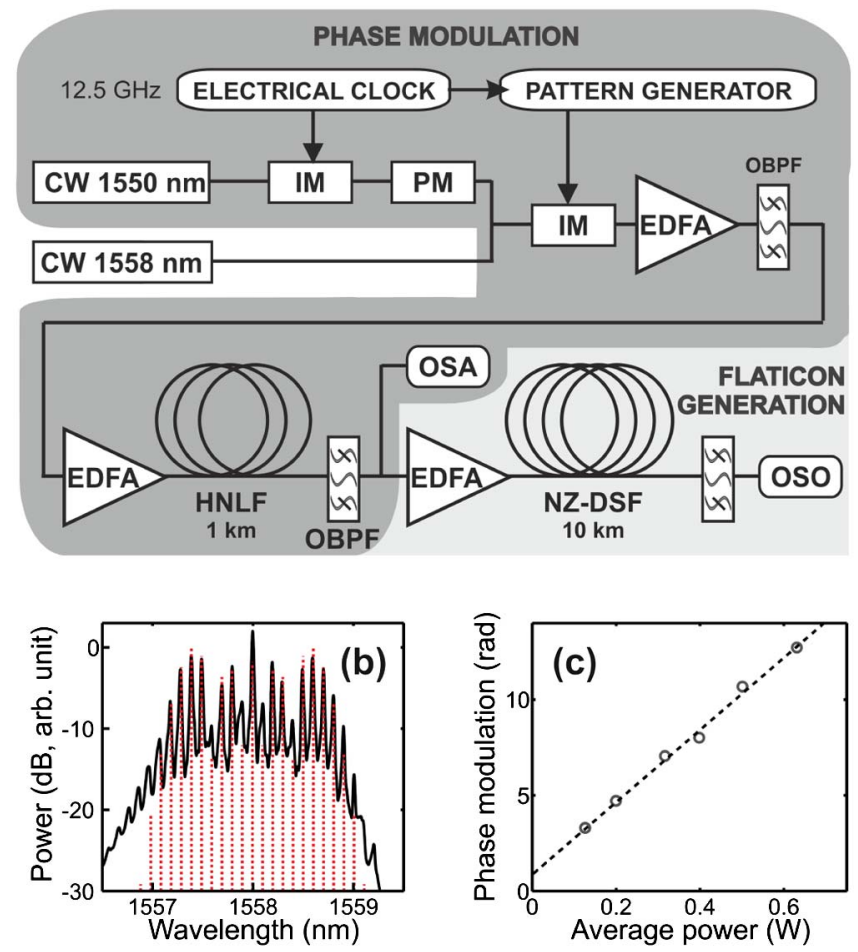

Fig. 1. (a) Experimental setup. (b) Optical spectrum of the continuous seed for an average pump power of $25 \mathrm{dBm}$. Experimental results (black solid line) are compared with analytical results of $\mathrm{CW}$ with a sinusoidal phase modulation of $7.3 \mathrm{rad}$ [gray-dashed line (red online)]. (c) Experimental evolution of the amplitude of the phase modulation according to the pump power in the HNLF. Experimental results (circles) are compared with a linear fit (gray-dashed line). devoted to make sure that the modulator is operated in the linear part of its transfer function, in order to maintain a purely sinusoidal modulation. The pump is also phase modulated at a low frequency (typically $100 \mathrm{MHz}$ ) in order to avoid any detrimental Brillouin backscattering during propagation. We have checked that such additional phase modulation of the pump has no influence on the phase modulated CW signal that is generated by the XPM process. Note that a second intensity modulator driven by a deterministic nonreturn to zero pattern of 10 successive ones followed by 22 zeros leads to packets of 800 ps duration repeating every $2.56 \mathrm{~ns}$. This enables to achieve, for a given average power, a more than threefold increase of the peak power. In order to compensate for the optical losses of the different modulators, an erbium-doped fiber preamplifier is inserted, followed by an adequate programmable optical bandpass filter (OBPF) that limits the building up of amplified spontaneous emission. A high power EDFA (HP EDFA) is then used to obtain the desired power level at the HNLF input.

In order to achieve a maximum XPM efficiency, the pump wave and the initial CW seed that is frequency shifted by $1 \mathrm{THz}$ (wavelength of $1558 \mathrm{~nm}$ ) relative to the pump wavelength co-propagate in polarization maintaining components up to the HNLF. At the output of the HNLF, the seed is spectrally isolated by an OBPF with a central frequency of $1558 \mathrm{~nm}$ and a spectral width of $400 \mathrm{GHz}$. An example of the experimentally generated spectrum broadened by XPM is provided in Fig. 1(b), where it can be compared with the theoretical spectrum of a CW that is sinusoidally phase modulated with an amplitude of $7.3 \mathrm{rad}$. We have checked using high-speed photodiodes and a sampling oscilloscope that no significant frequency modulation to amplitude modulation conversion affects the continuous seed during its propagation in the HNLF with low dispersion value. The excellent agreement between the experimental and the theoretical spectra validates the efficient transfer of the intensity modulation of the pump wave into a quasisinusoidal phase modulation of the $\mathrm{CW}$ seed at a frequency of $12.5 \mathrm{GHz}$. Figure 1(c) shows that a phase modulation as high as $12 \mathrm{rad}$ can be obtained for an average pump power of $0.6 \mathrm{~W}$. The linear fit of the dependence of the XPM-induced phase modulation amplitude as a function of pump power is fully consistent with the well-known laws of cross phase modulation.

The second part of the experimental setup relies on a HP EDFA followed by a $10 \mathrm{~km}$ long nonzero dispersion shifted fiber (NZ-DSF) with a GVD of $5 \mathrm{ps}^{2} / \mathrm{km}$ at $1550 \mathrm{~nm}$, a nonlinear coefficient of $1.7 \mathrm{~W}^{-1} \cdot \mathrm{km}^{-1}$, and a linear attenuation of $0.2 \mathrm{~dB} / \mathrm{km}$. The dispersion slope of the fiber is $0.066 \mathrm{ps}^{2} / \mathrm{km} / \mathrm{nm}$ and has been found to have a negligible influence. At the output of the fiber, an optical sampling oscilloscope (OSO, $500 \mathrm{GHz}$ bandwidth, EXFO PSO-100 series) with a picosecond time resolution was used to directly record the temporal intensity profile of the generated train of flaticon pulses.

Results obtained for three input signal power levels injected into the NZ-DSF are plotted in Fig. 2, and are compared with the corresponding expected results obtained from the numerical integration of the NLSE. The experimental results are in pretty good agreement 


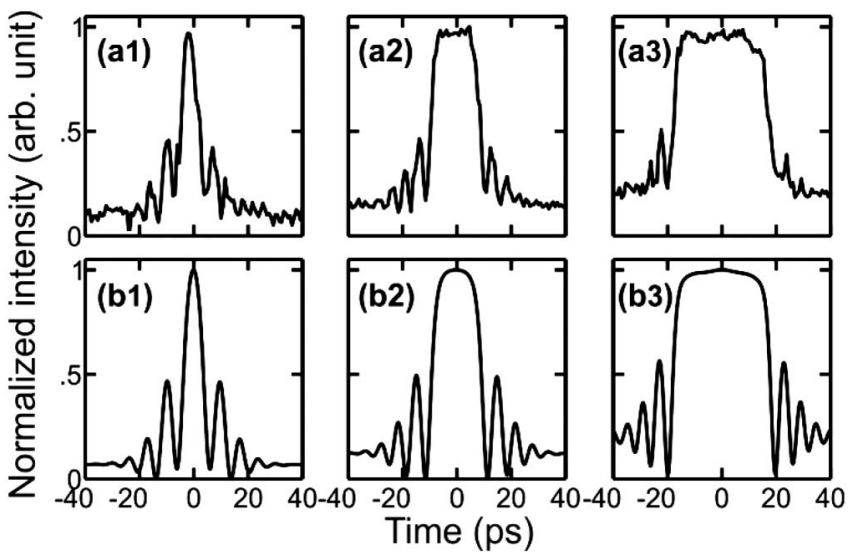

Fig. 2. Temporal intensity profiles at the output of the NZ-DSF obtained at a phase modulation of $6.75 \mathrm{rad}$ and at three different input powers: (1) $19 \mathrm{dBm}$, (2) $24 \mathrm{dBm}$ and (3) $28 \mathrm{dBm}$. Experimental results (subplots a) are compared with results of numerical integration of the NLSE (subplots b).

with the numerical predictions, and clearly exhibit several of the important features of optical flaticons. First, we may point out that pulses obtained at a sufficient power level clearly exhibit the remarkable flattened temporal intensity profile with strong time oscillating shock structures in the wings. The resulting nonlinear wave structure sits over a nonzero background: the peak power of the pulse is significantly higher than the background. The contrast of the experimental oscillations is however reduced when compared with the values expected from numerical simulations. We attribute this contrast reduction to the finite temporal resolution of the OSO as well as the influence of the residual noise induced by the three cascaded amplifiers in the setup. At low powers, the critical frequency $f_{c}$ is reduced so that $f_{s}$ exceeds $2.4 f_{c}$, and no stable flaticons can be generated.

A more systematic study of the dependence of the output temporal intensity profile as a function of the input signal power injected into the NZ-DSF is presented in Fig. 3. Such study provides a qualitative view of the longitudinal intensity evolution along the fiber. Hence the destructive cut-back method can be avoided. The
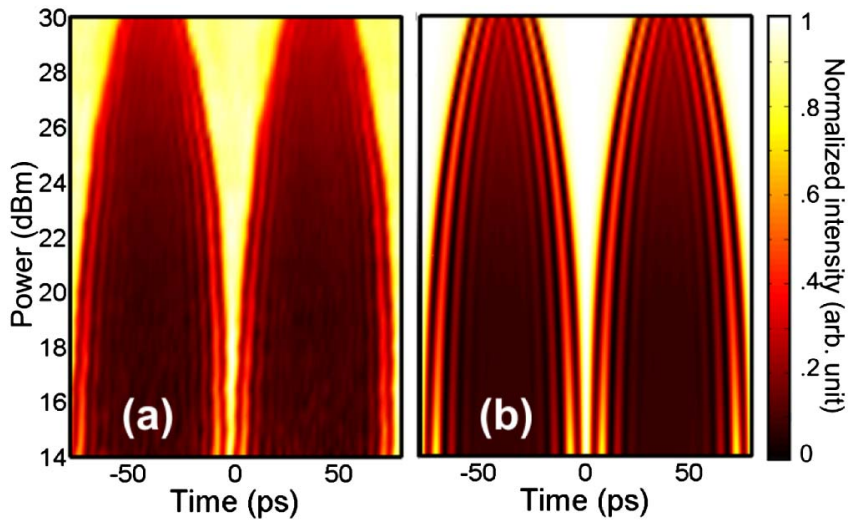

Fig. 3. Evolution of the temporal intensity profile as a function of the input average power injected into the NZ-DSF for a phase modulation of $6.75 \mathrm{rad}$. Experimental results (panel a) are compared with numerical simulations (panel b).
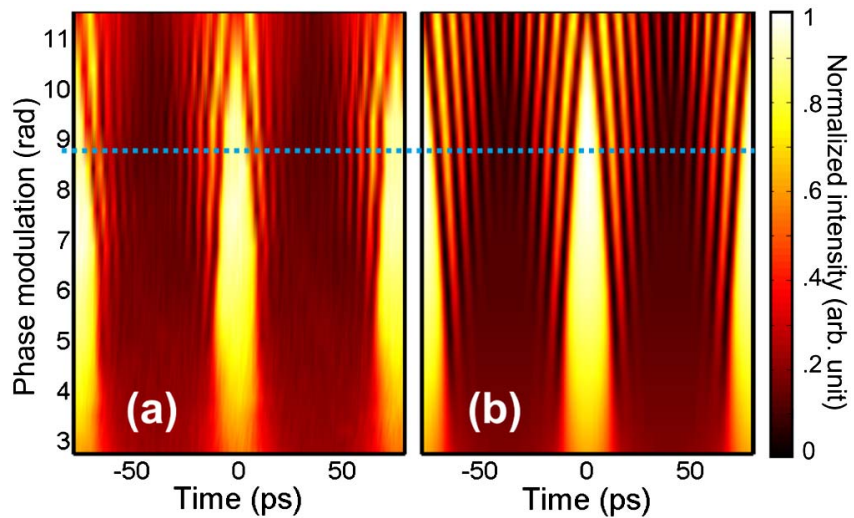

Fig. 4. Evolution of the temporal intensity profile as a function of the amplitude of the initial phase modulation. Experimental results (panel a) are compared with numerical simulations (panel b). The dotted line (blue online) denotes the phase amplitude leading to the frequency jump of $2.4 f_{c}$.

self-similar behavior of the central part of the flaticon is clearly observed in the experiments: Once again the observations are in close agreement with the numerical simulations summarized in Fig. 3(b). By changing the pump power injected into the HNLF, we have also investigated the influence of the initial level of phase modulation amplitude for a fixed signal power launched into the NZ-DSF set to $250 \mathrm{~mW}$. Different regimes can be observed, as outlined in Fig. 4. At low input phase modulation, the typical oscillations expected in the tails of the flaticon are not observed. For a phase modulation amplitude between 5 and $8 \mathrm{rad}$, clear signatures of flaticon pulses are revealed. For modulation amplitudes above $10 \mathrm{rad}$, the resulting frequency jump becomes too high, leading to an unstable pulse shape. This value can be linked to the threshold of $2.4 f_{c}$ that is estimated to $8.9 \mathrm{rad}$ in the absence of losses. All of these observations are fully consistent with the corresponding numerical simulations summarized in Fig. 4(b).

Finally, we have filtered the resulting output train of flaticons using an OBPF characterized by a Gaussian shape centered at the wavelength of the $\mathrm{CW}$ and a spectral bandwidth of $0.6 \mathrm{~nm}$. Results are plotted in
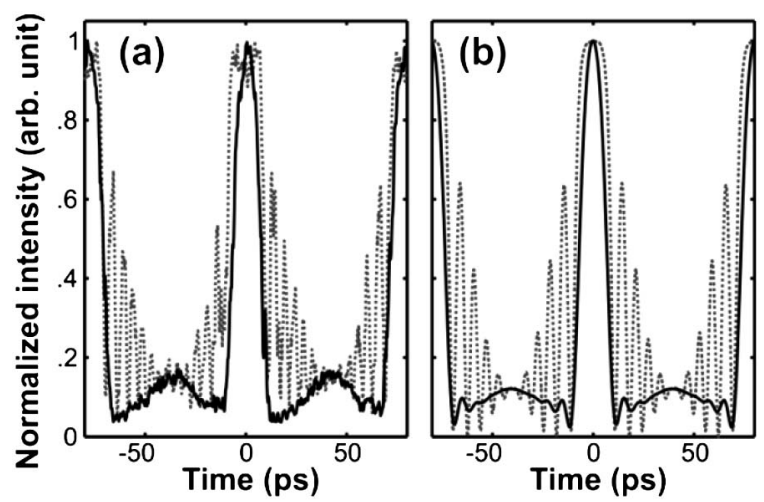

Fig. 5. Impact of a Gaussian OBPF of a flaticon pulse generated from an average signal power of $25 \mathrm{dBm}$ and an initial phase amplitude of $8 \mathrm{rad}$. The filtered pulse (solid black line) is compared with the flaticon pulse (gray-dotted line). Experimental results (panel a) are compared with numerical results (panel b). 
Fig. 5(a). As suggested in [14], this filtering removes the fast shock oscillations in the leading and trailing edges of the flaticons. However, as confirmed by the numerical simulations given in Fig. 5(b), a strong and long pedestal can be noticed between two successive filtered pulses. Consequently, the moderate resulting pulse train quality cannot compete at the present stage with other pulse train generation methods based on the nonlinear reshaping of an intensity modulated signal [17]. However, the interest of carrying out such a filtering is that it clearly demonstrates the chirp-free nature of the central part of the flaticon that remains quite unaffected by the filtering process. Such a feature also highlights the difference between a flaticon and a pulse that is obtained after propagation of a sinusoidally intensity modulated wave in a normally dispersive fiber, where the central part of the pulse structures becomes highly chirped [17].

To conclude, we have carried out the first experimental demonstration of the generation of flaticon pulses in a normally dispersive optical fiber. The nonlinear reshaping of an input phase modulated $\mathrm{CW}$ leads to the emergence of intense pulses with a flat top lying over a continuous background. The experimental results reveal strong shock oscillations in the edges of these pulses, as well as a self-similar evolution of the central part of the pulses. Our experiment confirms that an initial step-wise frequency modulation is not a mandatory requirement: Flaticon pulses can also emerge from a sinusoidal phase modulation. The reported generation of optical flaticons represents the key building block that may further permit for the observation of optical shallow water rogue waves [14]. Finally, we may expect that, similarly to the Peregrine solution, this research paves the way to new investigations in the field of hydrodynamics to help to understand the mechanism for the generation of sneaker waves.
We acknowledge the financial support of the Conseil Regional de Bourgogne (PARI Photcom), the iXCore Foundation and the funding of the Labex ACTION program (ANR-11-LABX-01-01), as well as the Agence Nationale de la Recherche (projects SO FAST and OPTIROC, ANR-11-EMMA-0005 and ANR-12-BS04-0011, respectively). The experimental work has benefited from the PICASSO Platform of the University of Burgundy. S. W. acknowledges support by Fondazione Cariplo (grant no. 2011-0395).

\section{References}

1. J. J. Stoker, Comm. Pure Appl. Math. 1, 1 (1948).

2. G. B. Whitham, Linear and Nonlinear Waves (Wiley, 1974).

3. J. E. Rothenberg and D. Grischkowsky, Phys. Rev. Lett. 62, 531 (1989).

4. V. I. Bespalov and V. I. Talanov, JETP Lett. 3, 307 (1966).

5. T. B. Benjamin and J. F. Feir, J. Fluid Mech. 27, 417 (1967).

6. A. Ritter, Z. Ver. Deutscher Ingen. 36, 947 (1982).

7. Y. Kodama and S. Wabnitz, Opt. Lett. 20, 2291 (1995).

8. P. Emplit, J. P. Hamaide, F. Reynaud, C. Froehly, and A. Barthelemy, Opt. Commun. 62, 374 (1987).

9. A. Chabchoub, O. Kimmoun, H. Branger, N. Hoffmann, D. Proment, M. Onorato, and N. Akhmediev, Phys. Rev. Lett. 110, 124101 (2013).

10. A. Slunyaev, I. Didenkulova, and E. Pelinovsky, Cont. Phy. 52, 571 (2011).

11. D. H. Peregrine, J. Austral. Math. Soc. Ser. B 25, 16 (1983).

12. K. Hammani, B. Kibler, C. Finot, P. Morin, J. Fatome, J. M. Dudley, and G. Millot, Opt. Lett. 36, 112 (2011).

13. I. Didenkulova and E. Pelinovsky, Nonlinearity 24, R1 (2011).

14. S. Wabnitz, C. Finot, J. Fatome, and G. Millot, Phys. Lett. A 377, 932 (2013).

15. S. Wabnitz, J. Opt. 15, 064002 (2013).

16. G. Biondini and Y. Kodama, J. Nonlinear Sci. 16, 435 (2006).

17. S. Pitois, C. Finot, J. Fatome, and G. Millot, Opt. Commun. 260, 301 (2006). 